Received May 25, 2017

Revised 1st, September 6, 2017 2nd, September 21, 2017 Accepted October 1, 2017

\section{Corresponding author}

Jeong Eun Lee, M.D.

Department of Anesthesiology and Pain Medicine, School of Medicine, Kyungpook National University, 680 Gukchaebosang-ro, Jung-gu, Daegu 41944, Korea

Tel: 82-53-420-5864

Fax: 82-53-426-2760

E-mail: pine253@hanmail.net

\section{ORCID}

http://orcid.org/0000-0002-7069-6264

\title{
Comparison of the incidence of venous thromboembolism between epidural and general anesthesia for total knee arthroplasty: a retrospective study
}

\section{Jeong Eun Lee', Dong-ho Jung', Jae-Min Yang' ', Won Kee Lee², Younghoon Jeon ${ }^{1,3}$, and Si-Oh Kim ${ }^{1}$}

${ }^{1}$ Department of Anesthesiology and Pain Medicine, ${ }^{2}$ Medical Research Collabration Center in KNUH, School of Medicine, Kyungpook National University, ${ }^{3}$ School of Dentistry, Kyungpook National University, Daegu, Korea

Background: Substantial variation exists in the reported rates of postoperative venous thromboembolism (VTE) following total knee arthroplasty (TKA) in the Asian population. This retrospective study aimed to compare the early postoperative VTE incidence between patients managed with epidural anesthesia and those managed by general anesthesia at the time of TKA.

Methods: We reviewed 589 cases of unilateral primary TKA performed between January 2011 and June 2014. We selected epidural versus general anesthesia groups as the main anesthetic choices, with postoperative patient-controlled analgesia. All the patients underwent deep vein thrombosis (DVT) computed tomography angiography on postoperative day 7 . The incidence of DVT and pulmonary thromboembolism (PTE) was evaluated and compared between epidural and general anesthesia.

Results: The overall incidence of VTE was $8.0 \%$ in the two groups together. The incidence did not differ between the groups. The odds ratio in the generalized estimation equations analysis showed a higher incidence of DVT and PTE in the epidural group; however, this result was not statistically significant. Although, the odds ratio for age showed that the risk of developing DVT and PTE increased 1.12 times per year.

Conclusions: Total VTE incidence was not significantly different between patients who underwent general anesthesia and those who underwent epidural anesthesia for TKA. A prospective multicenter study is required to evaluate the nature of the Korean VTE status in major orthopedic surgeries, and to prepare guidelines and protocols for medical prophylaxis for DVT and PTE in Korea.

Key Words: Epidural anesthesia, General anesthesia, Knee arthroplasty, Pulmonary embolism, Venous thrombosis.

\section{INTRODUCTION}

With increasing average life expectancy and the physical activeness of the population aged 60 and older, major ortho- pedic arthroplasty is becoming more common. In particular, total knee arthroplasty (TKA) has become a major orthopedic procedure in the aging population in Korea; additionally, venous thromboembolism (VTE) is one of the major compli-

This is an Open Access article distributed under the terms of the Creative Commons Attribution Non-Commercial License (http://creativecommons.org/licenses/by-nc/4.0) which permits unrestricted non-commercial use, distribution, and reproduction in any medium, provided the original work is properly cited. 
cations associated with TKA [1]. Substantial variation exists in the reported rates of postoperative VTE following TKA in the Asian population, with the general consensus being that the rates are lower in the Asian population than the Western population [1-4]. Over time, various studies have been published regarding the incidence of VTE following TKA, in the Korean population [3,5].

Major lower extremity orthopedic surgeries can be performed with either regional or general anesthesia. Previous studies have shown that regional anesthesia may decrease the incidence of deep vein thrombosis (DVT) and pulmonary thromboembolism (PTE) during major lower extremity orthopedic surgery $[6,7]$. Conversely, several previous studies also suggest a comparable incidence of VTE between regional and general anesthesia for total joint arthroplasty $[8,9]$. However, in case of general anesthesia, the predisposition to VTE could be offset with extensively applied postoperative pain control modalities and/or the newest technology. These could be used in conjugation with recently developed anesthetics for general anesthesia. Despite such varied results, until now, no study has compared the incidence of VTE due to regional versus general anesthesia.

The present study aims to compare the incidence of early postoperative VTE due to epidural versus general anesthesia for TKA. We hypothesize whether significant differences would exist between the two anesthetic methods.

\section{MATERIALS AND METHODS}

This retrospective study was approved by the Institutional Review Board of our hospital. Operative records from our hospital dated between January 2011 and June 2014 were reviewed. To determine the incidence of VTE according to anesthetic methods (epidural versus general), the criteria for exclusion was set as follows: previous VTE or venous disease, steroid or hormone replacement or oral contraceptive therapy, continuation of the anticoagulation or antiplatelet agent, congestive heart failure, respiratory failure, hematologic disease, malignancy, hip fracture, preoperative immobility, and a history of vascular surgery. These factors have been previously reported in the literature to be risk factors for VTE $[2,10,11]$. There were 589 cases of patients with primary TKA that met the inclusion criteria; patients with American Society of Anesthesiologists physical status I, or II comprised the study. A total of 208 patients had undergone total intravenous anesthesia (TIVA), whilst 381 patients had undergone continuous epidural anesthesia. The preoperative diagnosis of all patients in this study was limited to primary osteoarthritis. Prophylactic chemical anticoagulation for VTE was not used in case of both the groups. All operations included the use of a pneumatic tourniquet compression (Tourniquet 2500 ELC, VBM Medizintechnik GmbH, Germany), which was released before surgical skin closure. All patients wore calf-high intermittent pneumatic compression stockings on the contralateral leg, preoperatively, and a Jones compression dressing on the operated limb promptly after surgery. All patients were educated about post-operative exercises before the operation. Approximately 2 hours post-operatively, as soon as the patients recovered from anesthesia, active quadriceps and ankle motion exercises were initiated carefully while the patients were in bed. Two days after surgery, the drainage from the surgical site on the affected limb was removed, and the dressing was changed using a compression stocking. Active ambulation and continuous passive motion exercises were initiated if the patient's status of vital signs and wounds appeared to be normal. All drugs with antiplatelet activity were discontinued from 7 days before to 11 days after the operation until the international normalized ratio values returned to normal preoperative ranges. No other prophylactic antithrombotic drug was used during or after surgery. Our records showed that, epidural anesthesia was the main anesthetic choice, although, general anesthesia was performed according to the doctor's/patients' preferences, or in instances of expected procedural difficulties for epidural anesthesia, such as anatomical difficulties or previous history of spine surgery. Patients who had switched from epidural anesthesia to general anesthesia were not included in this study. Operation time was recorded from the first skin incision to the end of the last stitch of the wound. All patients were administered patient-controlled analgesia (PCA) to control postoperative pain and rehabilitate.

In the epidural anesthesia group (epidural group), ropivacaine (18-26 ml, 0.75\%) was administered through a lumbar epidural catheter. All patients received midline approach epidural anesthesia in the lateral decubitus position. The insertion area was $\mathrm{L}_{3}-\mathrm{L}_{4}$ or $\mathrm{L}_{4}-\mathrm{L}_{5}$ interspaces using the loss of resistance technique; the catheter was introduced $4-5 \mathrm{~cm}$ further above the insertion site. The upper level of analgesia 
(cold temperature test) extended, on average, to the fourth thoracic segment. We sedated the patients with $2 \%$ propofol, at $3-4 \mathrm{mg} / \mathrm{kg} / \mathrm{h}$, which was administered intravenously during surgery. The patients breathed $100 \%$ oxygen through a face-mask during the operation. After surgery, the patients were provided a mixture of $450 \mathrm{ml}$ of $0.25 \%$ ropivacaine and $50 \mu \mathrm{g}$ of fentanyl at a $2 \mathrm{ml} / \mathrm{h}$ flow rate, administered through the catheter for 2 days using epidural PCA (bolus dose $1 \mathrm{ml}$, lockout time 15 minutes).

In the general anesthesia group (general group), propofol was administered to the patients via a target-controlled infusion (TCI) device (Orchestra, Fresenius Vial, France). The effect-site concentration of propofol was titrated during the operation on the basis of the bispectral index within the range of 40-60. Remifentanil was simultaneously administered via TCI. The effect-site concentration of remifentanil was usually titrated within $20 \%$ variation, compared to pre-anesthetic vital signs that depended on the autonomic response, deduced through arterial pressure or heart rate during the operation. Rocuronium was used for tracheal intubation as well as intraoperative neuromuscular blockade. Intermittent positive pressure ventilation with an oxygen/air mixture was provided. After surgery, the patients were extubated and intravenous PCA with the mixture of fentanyl $(0.25 \mu \mathrm{g} / \mathrm{kg} / \mathrm{h})$ and ketorolac $(0.03 \mu \mathrm{g} / \mathrm{kg} / \mathrm{h})$ was used for 2 days (total volume including saline, $60 \mathrm{ml}$; basal rate, $0.5 \mathrm{ml} / \mathrm{h}$; bolus dose, 0.5 ml; lockout time, 15 minutes).

All the patients underwent routine DVT computed tomography (CT) with contrast medium, which was conducted for the lower extremity, the abdomen, and chest angiography on postoperative day 7 . The interpretation of the DVT CT angiography was conducted by the same experienced radiologist that conducted the procedure. Each lower extremity was scanned from the inguinal ligament to the level of the ankle, which included the trifurcation of the popliteal vein. The criteria for a proximal DVT was defined as a thrombus in the abdomen, femoral, or superficial femoral vein, whereas distal DVT was defined as a thrombus in the veins below the popliteal. PTE was diagnosed if a thrombus was seen in the segmental or lobar pulmonary artery.

In case a patient was diagnosed with proximal DVT or PTE, warfarin was immediately administered for VTE treatment based on the pulmonologist's consultation. An anticoagulant was administered until a follow-up DVT CT angiography was performed 3 months later.

During the postoperative period, we also reviewed for the occurrence of cardiopulmonary morbidity (hypotension, myocardial infarction, pulmonary edema), postoperative bleeding, opioid related adverse effect (nausea, vomiting, pruritus, sedation, urinary retention), cognitive defect, epidural anesthesia related neurologic complication, delayed motor blockade, or prolonged hospital stay, in addition to VTE.

Statistical analysis was performed using SAS 9.4 (SAS Institute Inc., USA). Descriptive statistics were expressed as mean \pm standard deviation for quantitative variables (age, height, weight, body mass index, and operation time) or frequency and proportion for categorical variables (gender, physical status, preoperative smoking, and VTE). The statistical significance of differences in mean values between two groups was assessed with a $t$-test or Mann-Whitney $U$ test as appropriate, and the associations between the developments of DVT and PTE were evaluated by $\chi^{2}$ test and Fisher's exact test (both DVT and PTE). Generalized estimating equations were used to discern correlations between differences (anesthetic group, age, and gender) and the binary responses (the incidence of VTE). The parameters were analyzed according to the anesthetic method (general vs. epidural), age (per year), and gender. These equations also generate odds ratios and $95 \%$ confidence intervals. The level of significance was set at $\mathrm{P}<0.05$ in all tests.

\section{RESULTS}

All surgeries were performed by the same surgeon. The cohort included 24 males and 184 females in the general group, and 48 males and 333 females in the epidural group. In each anesthesia group, the patients with VTE were significantly older than the ones in the no-VTE group. The other characteristics in the same anesthesia group showed no statistically significant difference relevant to the VTE diagnosis. All the demographic data of the patients in both the groups were statistically comparable except for the operation and anesthesia time. The operation time $(\mathrm{P}<0.001)$ and anesthesia time $(\mathrm{P}=$ 0.001 ) of the general anesthesia group was longer than that of the epidural anesthesia group; however, since the difference was within a 10 minute range, the difference is not likely to have clinical significance (Table 1). 
Table 1. Statistics for Demographic Data between Two Groups $(n=589)$

\begin{tabular}{|c|c|c|c|c|c|c|}
\hline \multirow[b]{2}{*}{ Parameter } & \multicolumn{3}{|c|}{ Epidural group } & \multicolumn{3}{|c|}{ General group } \\
\hline & $\begin{array}{c}\text { Total } \\
(\mathrm{n}=381)\end{array}$ & $\begin{array}{c}\text { No VTE } \\
(n=350)\end{array}$ & $\begin{array}{c}\text { VTE } \\
(n=31)\end{array}$ & $\begin{array}{c}\text { Total } \\
(\mathrm{n}=208)\end{array}$ & $\begin{array}{c}\text { No VTE } \\
(n=192)\end{array}$ & $\begin{array}{c}\text { VTE } \\
(n=16)\end{array}$ \\
\hline Age (yr) & $70.2 \pm 6.8$ & $69.7 \pm 6.7$ & $75.1 \pm 4.8^{*}$ & $71.0 \pm 6.9$ & $70.8 \pm 7.0$ & $74.3 \pm 5.7^{\dagger}$ \\
\hline Gender (M/F) & $48 / 333$ & $48 / 302$ & $0 / 31$ & $24 / 184$ & $23 / 169$ & $1 / 15$ \\
\hline Height (cm) & $154.6 \pm 10.6$ & $155.1 \pm 7.1$ & $154.0 \pm 5.2$ & $154.3 \pm 7.1$ & $154.5 \pm 7.1$ & $152.2 \pm 7.1$ \\
\hline Weight (kg) & $59.9 \pm 9.0$ & $59.9 \pm 9.1$ & $59.5 \pm 7.7$ & $59.8 \pm 9.5$ & $59.6 \pm 9.3$ & $62.1 \pm 11.7$ \\
\hline $\mathrm{BMI}\left(\mathrm{kg} / \mathrm{m}^{2}\right)$ & $24.8 \pm 3.5$ & $24.9 \pm 3.3$ & $25.1 \pm 3.0$ & $25.1 \pm 3.3$ & $24.9 \pm 3.2$ & $26.7 \pm 4.2$ \\
\hline ASA PS (I/II) & $54 / 327$ & $28 / 322$ & $26 / 5$ & $26 / 182$ & $25 / 167$ & $1 / 15$ \\
\hline Preoperative smoking & $19(5.0)$ & $17(4.9)$ & $2(6.5)$ & $10(4.8)$ & $9(4.7)$ & $1(6.3)$ \\
\hline Operation time (min) & $101.9 \pm 17.9$ & $102.1 \pm 18.4$ & $98.9 \pm 11.6$ & $111.3 \pm 30.6^{\ddagger}$ & $111.6 \pm 30.6$ & $107.8 \pm 30.5$ \\
\hline Anesthesia time ( $\mathrm{min}$ ) & $131.0 \pm 18.4$ & $131.2 \pm 18.9$ & $128.6 \pm 10.9$ & $138.0 \pm 27.1^{\S}$ & $138.1 \pm 27.6$ & $136.1 \pm 22.7$ \\
\hline
\end{tabular}

Values are presented as mean $\pm \mathrm{SD}$, number only or the number (\%). Epidural: the epidural anesthesia group, General: the general anesthesia group, VTE: venous thromboembolism, BMI: body mass index, ASA PS: American Society of Anesthesiologists physical status. Significant difference compared to No VTE in the same group: ${ }^{*} \mathrm{P}<0.001,{ }^{\dagger} \mathrm{P}=0.049$. Significant difference between the epidural group and the general group: ${ }^{\ddagger} \mathrm{P}<0.001$, ${ }^{\S} \mathrm{P}=0.001$.

Table 2. The Incidence of Venous Thromboembolism, Diagnosed on Day 7 Following Total Knee Arthroplasty, in both Groups

\begin{tabular}{|c|c|c|c|c|c|}
\hline Variable & Venous thromboembolism & Epidural group & General group & Total & P value \\
\hline No VTE & & $350(91.9)$ & $192(92.3)$ & $541(92.0)$ & \\
\hline \multirow[t]{6}{*}{ VTE } & & $31(8.1)$ & $16(7.7)$ & $47(8.0)$ & 0.849 \\
\hline & DVT only & & & & 0.182 \\
\hline & Proximal & $1(0.3)$ & $2(0.9)$ & $3(0.5)$ & \\
\hline & Distal & $15(3.9)$ & $12(5.8)$ & $27(4.6)$ & \\
\hline & PTE only & $4(1.0)$ & $1(0.5)$ & $5(0.9)$ & $0.661 *$ \\
\hline & DVT and PTE & $11(2.9)$ & $1(0.5)$ & $12(2.0)$ & $0.065 *$ \\
\hline Total & & $381(100)$ & $208(100)$ & $589(100)$ & \\
\hline
\end{tabular}

Values are presented as number (\%). VTE: venous thromboembolism, DVT: deep vein thrombosis, PTE: pulmonary thromboembolism. Proximal DVT means thrombus in the abdomen, femoral, or superficial femoral vein; distal DVT means thrombus in the veins below the popliteal; PTE means thrombus in the segmental or lobar pulmonary artery. *Fisher's exact test. P values $<0.05$ are regarded as statistically significant.

The overall incidence of VTE was $8.0 \%$ in the 589 cases of primary TKA. Although the surgery took longer in the general group, the incidence of DVT was comparable between the two groups. In contrast, there was a significantly higher incidence of PTE in the epidural group, compared to the general group. However, the incidence of both DVT and PTE did not differ between the two groups. One case of DVT was found in each group, where the DVT was detected on both the limbs. All the other patients found DVT on the operative limb. Location of thrombi was also similar in both the groups. Most cases of DVT were found in the calf vein (Table 2).

Generalized estimating equations analysis reported the odds ratio of VTE incidence depending on the anesthetic method, age, and gender; gender was found to have no statistical significance in determining the incidence. However, the odd ratio for age showed that the risk of developing VTE
Table 3. The Odds Ratio Analyzed by Generalized Estimating Equations for Group, Age, and Gender

\begin{tabular}{lcrrrrr}
\hline \multirow{2}{*}{ Parameter } & OR & \multicolumn{4}{c}{$95 \% \mathrm{Cl}$} & \\
\cline { 3 - 5 } & & Lower & Upper & & \\
\hline Epidural vs. General & 1.55 & 0.80 & 3.01 & 1.31 & 0.190 \\
Age (yr) & 1.12 & 1.07 & 1.17 & 5.01 & $<0.001$ \\
Female vs. Male & 7.32 & 0.99 & 54.28 & 1.95 & 0.051 \\
\hline
\end{tabular}

OR: odds ratio, $\mathrm{Cl}$ : confidence interval, Epidural: the epidural anesthesia group, General: the general anesthesia group. A continuous variable, patient age was examined per year. $P$ values $<0.05$ are regarded as statistically significant.

increased 1.12 times per year's increase in patient age (Table $3)$.

In our records, none of the patients had postoperative clinical symptoms of DVT or PTE, and no specific complications after TKA were observed. 


\section{DISCUSSION}

In studies investigating the western population, DVT after a major orthopedic surgery is known to occur in $40 \%-84 \%$ of patients without prophylaxis [12,13]. In Korea, the risks of developing of DVT and PTE following TKA are reported to be very low compared to those of patients from western countries $[1,3,5,14]$. The low incidence of VTE in Asians is thought to be due to differences in basic genetic factors, in addition to other risk factors such as low incidence of venous disease, obesity, and hyperlipidemia [15]. Although many studies have investigated the risk factors for VTE following TKA, most of them have used orthopedic approaches. In addition, no observation study in Korea has investigated VTE incidence depending on the type of anesthesia. If the influence of anesthesia on VTE incidence following TKA is identified, and early detection of VTE by a safe, reliable, and time-saving method is achievable, it may be helpful to prevent fatal PTE without the use of prophylactic anticoagulants.

Epidural anesthesia is the preferred anesthetic management for TKA $[6,16]$. It is considered to be superior in reducing blood loss, tourniquet pain, and analgesia, and the decreased incidence of VTE as a result of using epidural anesthesia is considered to be one of the reasons why epidural anesthesia is chosen over general anesthesia for TKA. Recent systematic reviews and meta-analysis studies have shown that although patient outcomes support regional anesthesia over general anesthesia during hospitalization, it has not been definitely concluded that there is a causal link between a particular anesthetic method and the improvement in the postoperative outcome for TKA $[17,18]$. In our results, we found no differences in the incidence of VTE, compared between epidural and general anesthesia for TKA. We postulate that this is mainly due to the technical development of general anesthesia and sufficient postoperative pain control for accelerated rehabilitation.

In our study, general anesthesia was performed with TIVA, and maintained with propofol and remifentanil using TCI. Propofol suppresses stress-related hormones and the inflammatory reaction, and inhibits platelet aggregation under surgical stimuli. The neuroendocrine stress response is caused by surgical stimulation that leads to hypercoagulability, especially during general anesthesia, when the concentration of catecholamine, cortisol, and growth hormones in the blood increase in response to the inhalation anesthetics [19-21]. These stress hormones have been shown to elevate platelet responsiveness to collagen, adenosine diphosphate, and ristocetin in humans [22]. Propofol causes the suppression of platelet aggregation via aforementioned mechanisms. Moreover, remifentanil has a very short duration of action, even when it is administered for a long duration. The anesthesia depth of remifentanil can be quickly controlled to minimize hemodynamic changes with the use of a tourniquet. In our study, all patients in the general group were monitored using the bispectral index for anesthetic depth, and anesthetics were administered via the TCI device. Because remifentanil has detrimental effects on hemodynamics if overdose, TCI device allows adequate infusion depending on pharmacokinetic models. This general anesthesia strategy prevents the synergistic effect of propofol, and allows the maintenance of stable cardiopulmonary function during the perioperative period.

TIVA contributes to early ambulation due to a rapid recovery from anesthesia, and is additionally advantageous in terms of avoiding potential complications, such as postoperative nausea and vomiting, and the fast recovery of cognitive function, which makes patients return to daily activities as soon as possible [23]. TIVA also has an advantage in terms of its analgesic effect on postoperative pain [20,24]. Because of the nature of TKA, which is a surgical treatment for osteoarthritis, elderly patients account for the majority of patients. Therefore, TIVA would be more beneficial than volatile inhalation anesthesia for accelerated rehabilitation of elderly patients who have undergone surgery.

In TKA, the tourniquet has the advantage of securing a surgical field, although, there is concern that it may increase the incidence of DVT after surgery. The location of most DVTs, located using a tourniquet, is calf veins, and the DVT location of our data showed an identical tendency. Distal DVT rarely causes fatal thromboembolic event such as symptomatic PTE, compared to proximal DVT, and mostly resolves spontaneously without any event [14]. We should also consider the effect of tourniquet time or operation time on DVT between the two groups. In a study by Hernandez et al. [25], they have not shown any relationship between tourniquet time and incidence of DVT, and the incidence of proximal DVT increased as the operation time was longer than 120 minutes. Although we could not obtain information about tourniquet 
time, the low DVT incidence in our study is presumed to be attributed to a short operation time that was less than 120 minutes, in both the groups.

We routinely delivered PCA for both groups after TKA. According to the type of anesthesia, the PCA application also differs; intravenous PCA is conducted in the general group and continuous epidural PCA is conducted in the epidural group. PCA enabled the patients of both the groups to achieve early ambulation and perform passive exercise under more comfortable postoperative conditions. Generally, postoperative pain control with epidural PCA is effective for a rapid recovery of exercise in orthopedic surgery, and intravenous opioid analgesics are likely to be adverse due to opioidrelated side effects and insufficient pain control for TKA [26]. However, the use of non-opioid analgesics as adjuvants can reduce the total dose of opioids in intravenous PCA, which improves postoperative analgesia [23]. Opioids have various side effects such as nausea, vomiting, ileus, pruritus, or confusion, and high doses of opioids would lead to severe side effects such as respiratory depression. Another concern regarding opioids is their intraoperative infusion, which may cause opioid-induced hyperalgesia during the postoperative period. To overcome the aforementioned side effects of opioids in our patients, non-steroidal anti-inflammatory drugs (NSAID) were included in intravenous PCA, and intravenous bolus $50 \mu \mathrm{g}$ of fentanyl and intravenous PCA was administered simultaneously 10 minutes before emergence to enhance the quality of analgesia during recovery. Thus, there were no patients with complications related to intravenous PCA. Although there is evidence that NSAIDs increases the risk of VTE [27], we are currently unsure if NSAIDs or other confounding factors increase the risk of VTE.

Brooks et al. [28] showed a 3.8\% of overall incidence of VTE following TKA, with epidural anesthesia. These authors used sonography to diagnose VTE. This incidence is much lower than our results of $7.1 \%$ incidence of DVT alone and $8.0 \%$ incidence of both DVT and PTE. Kim and Kim [14] reported an overall 26.6\% DVT incidence in Korea. These studies imply that the incidence of VTE shows a wide variation according to the diagnostic modalities used, whether or not chemoprophylaxis was provided and when the diagnostic test was performed.

Among the imaging modalities used to diagnose postoperative DVT, venography and color Doppler ultrasonography have been commonly used for the early detection of DVT. However, these techniques have crucial disadvantages [29]. Venography is highly useful for DVT detection, although, not for PTE detection, and the procedure itself might cause allergic reactions and DVT occurrence; additionally, it is highly invasive. When using color Doppler ultrasonography, patients need to change positions, such as supine and prone positions. Using this diagnostic tool, it is also difficult to accurately examine the lesions in the inferior vena cava, iliac vein, and the pulmonary vein. Despite a high specificity, the sensitivity of ultrasound for detecting asymptomatic DVT is low (47\%-62\%) and has low inter-observer reproducibility [29]. Also, ultrasonography is unable to diagnose PTE $[29,30]$ and its sensitivity for detecting DVT after total joint arthroplasty is low.

In our study, we used DVT CT, a highly sensitive diagnostic tool for postoperative VTE diagnosis. DVT CT angiography takes a relatively short time to perform without positional changes, and can examine the lower limb vessels, including the regions of the thoracic, abdominal, and pelvic vessels [30]. This modality is highly sensitive and specific for diagnosing DVT. In addition, asymptomatic DVT can be detected early, and the difference between VTE incidence depending on the anesthetic method can be observed more clearly.

Despite presenting a lack of significant difference in the incidence of VTE between general and epidural anesthesia, our study has certain limitations. First, we did not perform preoperative VTE screening. Our results might include patients with preoperative VTE, despite having no preoperative symptomatic evidence for DVT or PTE in our review. Second, this study mainly focused on VTE complications; although, other complications during TKA should simultaneously be evaluated, including long-term mortality. VTE is one of a range of possible complications that occur as a result of TKA. An integrated consideration of other complications could provide a more sophisticated approach to the anesthetic management used for TKA. Third, the very low incidence of VTE or PTE might distort the comparative interpretation of our results to those of previous studies. In conclusion, the correlation between the factors could be confirmed in the retrospective study, but we could not infer exact conclusions about causality from the retrospective data. Thus, a planned prospective study with a larger sample size should be enabled through a multicenter study. This type of project will provide more 
accurate data to evaluate the exact incidence of DVT and prepare guidelines and protocols for medical prophylaxis, none of which exist in the Asian population.

In summary, there was no statistical difference in the incidence of VTE after TKA between patients receiving general anesthesia using TIVA and those receiving epidural anesthesia in our study. Therefore, if we could demonstrate that discreet techniques of general anesthesia, and epidural anesthesia have a similar effect against VTE with early diagnosis using DVT CT, it would help to establish guidelines and protocols for anesthetic and analgesic techniques for VTE prevention following TKA in Korea.

\section{REFERENCES}

1. Kim YJ, Hur CI, Song EK, Seon JK, Park SJ, Cho SB, et al. Availability of D-dimer test for the diagnosis of deep vein thrombosis after total knee arthroplasty. J Korean Orthop Assoc 2007; 42: 523-9.

2. Geerts WH, Pineo GF, Heit JA, Bergqvist D, Lassen MR, Colwell CW, et al. Prevention of venous thromboembolism: the Seventh ACCP Conference on Antithrombotic and Thrombolytic Therapy. Chest 2004; 126(3 Suppl): S338-400

3. Kim YH. The incidence of deep vein thrombosis after cementless and cemented knee replacement. J Bone Joint Surg Br 1990; 72 : 779-83.

4. McKenna R, Bachmann F, Kaushal SP, Galante JO. Thromboembolic disease in patients undergoing total knee replacement. J Bone Joint Surg Am 1976; 58: 928-32.

5. Byun SS, Kim JH, Kim YJ, Jeon YS, Park CH, Kim WH. Evaluation of deep vein thrombosis with multidetector row CT after orthopedic arthroplasty: a prospective study for comparison with Doppler sonography. Korean J Radiol 2008; 9: 59-66.

6. Rodgers A, Walker N, Schug S, McKee A, Kehlet H, van Zundert A, et al. Reduction of postoperative mortality and morbidity with epidural or spinal anaesthesia: results from overview of randomised trials. BMJ 2000; 321: 1493.

7. Sharrock NE, Haas SB, Hargett MJ, Urquhart B, Insall JN, Scuderi G. Effects of epidural anesthesia on the incidence of deep-vein thrombosis after total knee arthroplasty. J Bone Joint Surg Am 1991; 73: 502-6.

8. Helwani MA, Avidan MS, Ben Abdallah A, Kaiser DJ, Clohisy JC, Hall BL, et al. Effects of regional versus general anesthesia on outcomes after total hip arthroplasty: a retrospective propensitymatched cohort study. J Bone Joint Surg Am 2015; 97: 186-93.

9. Memtsoudis SG, Sun X, Chiu YL, Stundner O, Liu SS, Banerjee S, et al. Perioperative comparative effectiveness of anesthetic technique in orthopedic patients. Anesthesiology 2013; 118: 1046-58.

10. Yoo MC, Cho YJ, Ghanem E, Ramteke A, Kim KI. Deep vein thrombosis after total hip arthroplasty in Korean patients and Ddimer as a screening tool. Arch Orthop Trauma Surg 2009; 129: 887-94.

11. Tan M, van Rooden CJ, Westerbeek RE, Huisman MV. Diagnostic management of clinically suspected acute deep vein thrombosis. Br J Haematol 2009; 146: 347-60.

12. Stringer MD, Steadman CA, Hedges AR, Thomas EM, Morley TR, Kakkar VV. Deep vein thrombosis after elective knee surgery. An incidence study in 312 patients. J Bone Joint Surg Br 1989; 71: 492-7.

13. Stulberg BN, Insall JN, Williams GW, Ghelman B. Deep-vein thrombosis following total knee replacement. An analysis of six hundred and thirty-eight arthroplasties. J Bone Joint Surg Am 1984; 66: 194-201.

14. Kim YH, Kim JS. Incidence and natural history of deep-vein thrombosis after total knee arthroplasty. A prospective, randomised study. J Bone Joint Surg Br 2002; 84: 566-70.

15. Kim YH, Yoo JH, Kim JS. Factors leading to decreased rates of deep vein thrombosis and pulmonary embolism after total knee arthroplasty. J Arthroplasty 2007; 22: 974-80.

16. Perka C, Arnold U, Buttgereit F. Influencing factors on perioperative morbidity in knee arthroplasty. Clin Orthop Relat Res 2000; (378): 183-91

17. Johnson RL, Kopp SL, Burkle CM, Duncan CM, Jacob AK, Erwin PJ, et al. Neuraxial vs general anaesthesia for total hip and total knee arthroplasty: a systematic review of comparative-effectiveness research. Br J Anaesth 2016; 116: 163-76.

18. Macfarlane AJ, Prasad GA, Chan VW, Brull R. Does regional anesthesia improve outcome after total knee arthroplasty? Clin Orthop Relat Res 2009; 467: 2379-402.

19. Mendez D, De la Cruz JP, Arrebola MM, Guerrero A, GonzálezCorrea JA, García-Temboury E, et al. The effect of propofol on the interaction of platelets with leukocytes and erythrocytes in surgical patients. Anesth Analg 2003; 96: 713-9.

20. Lee WK, Kim MS, Kang SW, Kim S, Lee JR. Type of anaesthesia and patient quality of recovery: a randomized trial comparing propofol-remifentanil total i.v. anaesthesia with desflurane anaesthesia. Br J Anaesth 2015; 114: 663-8.

21. Gilliland HE, Armstrong MA, Carabine U, McMurray TJ. The choice of anesthetic maintenance technique influences the antiinflammatory cytokine response to abdominal surgery. Anesth Analg 1997; 85: 1394-8.

22. Rosenfeld BA, Faraday N, Campbell D, Dise K, Bell W, Goldschmidt P. Hemostatic effects of stress hormone infusion. Anes- 
thesiology 1994; 81: 1116-26.

23. Buvanendran A, Reuben SS, Kroin JS. Recent advances in nonopioid analgesics for acute pain management. Tech Reg Anesth Pain Manage 2007; 11: 19-26.

24. Kim YS, Lee WK, Choi YS, Chae YK, Ahn SW, Lee A, et al. A comparison of the recovery characteristics of propofol-remifentanil and desflurane-remifentanil anesthesia under bispectral index (BIS) monitoring following laparoscopic cholecystectomy. Anesth Pain Med 2011; 6: 331-5.

25. Hernandez AJ, Almeida AM, Fávaro E, Sguizzato GT. The influence of tourniquet use and operative time on the incidence of deep vein thrombosis in total knee arthroplasty. Clinics (Sao Paulo) 2012; 67: 1053-7.

26. Ranawat AS, Ranawat CS. Pain management and accelerated rehabilitation for total hip and total knee arthroplasty. J Arthro- plasty 2007; 22(7 Suppl 3): 12-5.

27. Huerta C, Johansson S, Wallander MA, García Rodríguez LA. Risk factors and short-term mortality of venous thromboembolism diagnosed in the primary care setting in the United Kingdom. Arch Intern Med 2007; 167: 935-43.

28. Brooks PJ, Keramati M, Wickline A. Thromboembolism in patients undergoing total knee arthroplasty with epidural analgesia. J Arthroplasty 2007; 22: 641-3.

29. Segal JB, Eng J, Tamariz LJ, Bass EB. Review of the evidence on diagnosis of deep venous thrombosis and pulmonary embolism. Ann Fam Med 2007; 5: 63-73.

30. Dronkers CE, Klok FA, Huisman MV. Current and future perspectives in imaging of venous thromboembolism. J Thromb Haemost 2016; 14: 1696-710. 